\title{
Prediction of Field Flow and Temperature Profile in Calandria Vessel
}

\author{
Ankul Pratap Singh ${ }^{1}$, Dr. M. D. Nadar ${ }^{2}$ \\ ${ }^{1}$ Mumbai University, Pillai Institute of Information Technology, Engineering, Media Studies \& Research, \\ Sector 16, New Panvel 410206, India \\ ${ }^{2}$ Pillai Institute of Information Technology, Engineering, Media Studies \& Research \\ Sector 16, New Panvel 410206, India
}

\begin{abstract}
Nuclear power generator consists of nuclear reactor, steam generator, heavy water turbine, condenser, and hot well pump. Nuclear reactor generates steam at high-pressure 70 bar to 100 bar and temperature range $400 \mathrm{~K}$ to $525 \mathrm{~K}$. It consists of heavy water which acts as moderator. The moderator has fluid properties of velocity, viscosity, density, temperature, pressure etc. Moderator is a medium that reduces the speed of fast neutrons, turning them into thermal neutrons capable of sustaining a nuclear chain reaction. Due to this, moderator gets heated and removes heat from nuclear reactor core. The problems faced in nuclear reactor are as follows, nuclear chain reaction is happening in the core of the reactor. During chain reaction, core emits neutrons and large amount of atomic energy. The generated atomic energy converts coolant and heavy water into steam with high pressure and temperature. The high pressure and high temperature of generated steam may lead to burst the nuclear reactor. This paper includes identifying the technical parameters, which are involved in the nuclear reactor chain reactions. The technical parameter intensities are to be measured and are need to take necessary safety precautionary measurements to protect abnormal accidents in the nuclear reactor. Technical process parameters of generated steam like pressure, temperature, energy and velocities etc. are been considered in this investigation for safety precautionary measures. This paper gives a relation amongst temperature, pressure, velocity and energy; mathematical model is used to predict the temperature and velocity at particular instant and position of fluids.
\end{abstract}

Keywords: Prediction of field flow, Temperature Profile, Calandria Vessel, Pressure profile, Fluent

\section{Introduction}

Nuclear power generation is based on rankine cycle. Rankine cycle consist of process of pressure addition in pump house, heat addition in boiler, work developed in steam turbine and heat rejection in condenser. Advanced nuclear reactor uses uranium as a fuel and goes through number of chain reactions. Uranium-fuelled nuclear power is an efficient and clean way of boiling water to make steam; Nuclear reactor generated steam drives turbines. Nuclear reactor works like most gas-fired power stations or coal. Fuel is made up of heavy atoms that split when they absorb neutrons. Fuel is placed into the reactor vessel (basically a large tank) along with a small neutron source. Nuclear reactor uses coolant and moderator in the nuclear chain reaction process. Coolant is removing the thermal heat energy from nuclear chain reaction and converts coolant into steamin which the generated steam to drive steam turbine shaft. Nuclear fission is a nuclear reaction in which the nucleus of an atom breaks into tiny parts and producing free neutrons and photons (in the form of gamma rays). The key to maintaining a nuclear reaction within a nuclear reactor is to use the neutrons being released during fission to stimulate fission in other nuclei. It is also known as chain reaction because it sustain with self-control over the geometry and reaction rates. Function of moderator in the nuclear reactor is a medium that reduces the speed of fast neutrons, converting them into thermal neutrons, which are capable of controlling a nuclear chain reaction involving ${ }^{235} \mathrm{U}$ in the reactor. Commonly used moderators are heavy water solid graphite, water (light) in the nuclear reactor. Hydrocarbons, beryllium have also been used as moderator in the nuclear reactor. Neutrons are generally bound into an atomic nucleus, and do not sustain free for long in nature. The unbound neutron has a small half-life of just less than 15 minutes. The release of neutrons from the nucleus needs exceeding the binding energy of the neutron, which is typically 7-9 MeV. Whatever the source of neutrons, they are released with energies of some $\mathrm{MeV}$. Water makes an excellent moderator. Heavy water is used as moderator in nuclear reactor. Heavy water contains a more proportion of the hydrogen isotope i.e. deuterium, either as deuterium oxide, $\mathrm{D}_{2} \mathrm{O}$ or $\mathrm{H}_{2} \mathrm{O}$ water, physically and chemically, it resembles water, the deuterium-to- hydrogen ratio is about $156 \mathrm{ppm}$.Fluid (coolant) flows through core of nuclear reactor get converted into super-heated steam (high temperature, high pressure) and super-heated steam passes through steam turbine to drive turbine shaft.

Coolant and moderator flows through the nuclear reactor core. Coolant to absorb thermal energy in the nuclear chain reaction process and moderator controls high rate of speed of nuclear chain reaction process neutrons. Nuclear reactor consists of coolant and moderator two fluids. Coolant carries the thermal and pressure energy in the form of steam from nuclear core to steam turbine and moderator is used to control the fast neutron speed. In this project, investigation of operating parameter of moderator fluid like velocity, temperature, pressure and energy are carried out.

In this paper to investigate and analyze moderator thermal and fluid properties, when it flows in the vessel of advanced water nuclear reactor. This investigation and analysis of moderator thermal and fluid properties to predict heat transfer quantity of nuclear chain reaction inside and around calandria tubes and tanks. This investigation and analysis suggests taking necessary safety precautionary measures in the nuclear reactor chain reaction process. [1] 


\section{International Journal of Science and Research (IJSR) \\ ISSN (Online): 2319-7064 \\ Index Copernicus Value (2013): 6.14 | Impact Factor (2014): 5.611}

The following properties of moderator pressure, temperature, velocity, energy and type of moderator flow are considered in nuclear reactor chain reaction process to develop and establish relation amongst moderator properties like temperature, velocity, pressure and energy in the form of mathematical model by using student version CFD software ANSYS FLUENT. Mathematical model is also developed to predict the temperature and velocity at particular instant and position of fluids. Using FLUENT software and taking SIMPLE scheme for laminar flow model, simulation carried out. SIMPLE mathematical model stands for Semi Implicit Method for Pressure Linked Equation.

\section{Literature Review}

The Indian nuclear power program has been conceived bearing in mind that the optimum utilization of domestic thorium and uranium reserves with the objective of providing long-way energy security to the country. One of the needful elements of the Indian strategy is to increase the quality of the fuel utilization using a closed fuel cycle. This entails reprocessing of the spent fuel to recover fissile and fertile materials and its recycle back into the system. Considering this objective, the indigenous nuclear power program in India was initiated with Pressurized Heavy Water Reactors (PHWRs) using natural uranium and heavy water, and based on pressure tube technology. The pressure tube concept, used in PHWRs, has several advantages such as a high conversion ratio with well thermal energy acquired neutron spectrum due to cold moderator, physical separation of the high-temperature high-pressure coolant from the lowtemperature low-pressure moderator; low excess reactivity in the core arising out of on-power fuelling a greater flexibility in adopting different refueling schemes. [3]

India has been operating and developing improved versions of its current generation PHWRs on the basis of international trends, operating experience and indigenous R\&D inputs as a first stage. In the second stage of the Indian nuclear power program, plutonium from the natural uranium-based PHWRs will be used in FBR(Fast Breeder Reactors) for multiplying the fissile reaction.[4] Considering the large thorium reserves in India, the future systems, in the third stage of Indian nuclear power program, will be depend on thorium- ${ }^{233} \mathrm{U}$ fuel cycle. While the initiation of the third stage, it will take place in the future; there is a requirement for the timely development of thorium-based technologies for the complete thorium fuel cycle. The Advanced reactor is being developed to fulfill this need.

Advanced water reactor is a $300 \mathrm{MWe}$, vertical pressure tube (PT) type, boiling light water cooled, and heavy water moderated reactor. The reactor integrates a number of passive safety structures and is related with a fuel cycle having concentrated environmental impact. In case of serve accident condition, such as loss of coolant accident, the fuel bundles are devoid of coolant \& it tends to get heated up. The nuclear fuel bundles are housed in pressure tube, which is housed again in the Calandria tube. The temperature of the fuel pins rises $\&$ heat transfer become dominant [5]. This study of accident scenario is also been studied in this work. This work is carried out where it employs natural circulation system for cooling the reactor core under shutdown and operating conditions. The Main Heat Transport (MHT) System transports heat from fuel pins to steam drum by boiling light water as the coolant.

\section{Methodology}

To identify the safety problem of nuclear calandria vessel from previous research work, journals, etc. In this project, to identify the problems, to collect technical parameter of moderator, to affect the safety performance of nuclear calandria vessel, to develop the mathematical model by establishing relation among technical parameter with the help of momentum, energy and continuity equations.

Moderator flow in calandria tank is analyzed by using ANSYS FLUENT software to analyze the mathematical model of moderator flow in the calandria tank. GAMBIT is modeling software, which is used for developing geometry and mesh of the calandria tank in 2D and 3D modeling.

The dimensions of the calandria tank are below

- Diameter of Calandria Tube (CT) $(\mathrm{m})=0.0889$

- Diameter of calandria vessel $(\mathrm{m})=0.500$

- Diameter of first layer of CT $(\mathrm{m})=0.120$

- Diameter of second layer $(\mathrm{m})=0.240$

- Diameter of inlet and outlet $(\mathrm{m})=0.05$

- Height of Calandria Vessel $(\mathrm{m})=0.600$

- Inlet height from bottom $(\mathrm{m})=0.1$

- Outlet height from bottom $(\mathrm{m})=0.5$

- Length of inlet and outlet pipes $(\mathrm{m})=0.1$

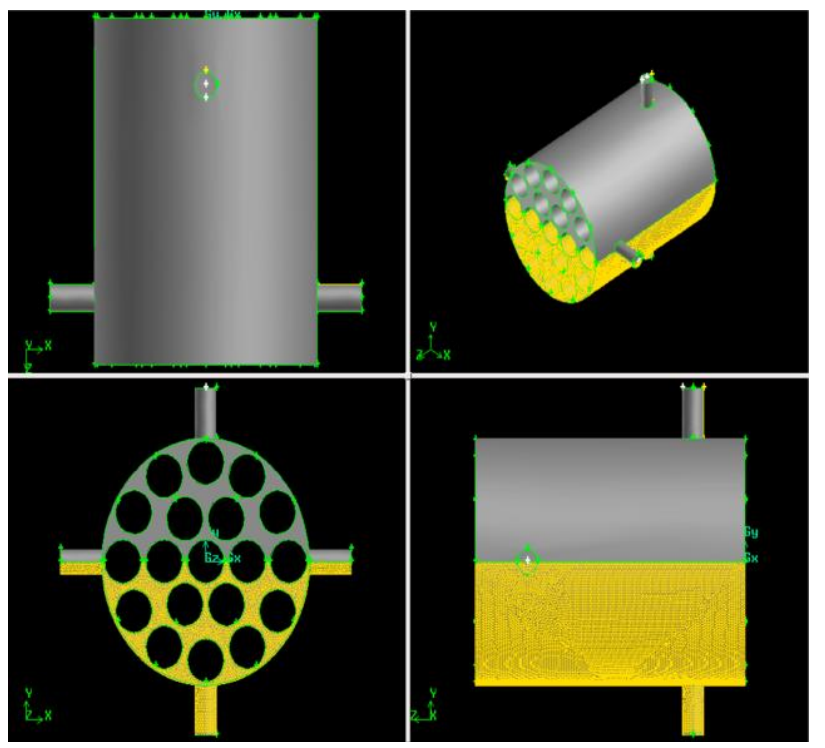

Figure 3.1: Calandria Vessel

Calandria vessel is cylindrical vessel made up SS304L. It is also called as moderator tank. There are 19- calandria tubes having $88.9 \mathrm{~mm}$ dia each and length of tube is $600 \mathrm{~mm}$. Calandria tubes are arranged in a circular periphery pattern in the number of 1, 6 and 12 (Fig. No. 3.1) that is there are three concentric circles with diameter of $0,120 \mathrm{~mm}$ and 240 $\mathrm{mm}$. At the center there is single tube then first layer of 120 $\mathrm{mm}$ dia has 6 tubes and second layer of $240 \mathrm{~mm}$ dia has 12 tubes. In each calandria tube there is a pressure tube and uranium is fueled in this pressure tube. Space $(5 \mathrm{~mm})$ 


\section{International Journal of Science and Research (IJSR) \\ ISSN (Online): 2319-7064}

Index Copernicus Value (2013): 6.14 | Impact Factor (2014): 5.611

between calandria tube and pressure tube is filled with Helium noble gas.

Heavy water $\left(\mathrm{D}_{2} \mathrm{O}\right)$ is used as moderator, which is filled inside of calandria tank. There are two inlets of $50 \mathrm{~mm}$ dia, above $100 \mathrm{~mm}$ from the bottom end and opposite to each other and also there are two outlets of $50 \mathrm{~mm}$ dia below $100 \mathrm{~mm}$ from top end of the vessel. Pipe length of inlet and outlet is same i.e. is $100 \mathrm{~mm}$.

\section{Result \& Analysis}

Moderator flow in calandria vessel of advance nuclear reactor is simulated using surface heating method. Experimental setup of advance water nuclear reactor model with specification of $3.5 \mathrm{KW}$ consist of 19 calandria tubes, 500 fuel channels, calandria vessel, coolant and moderator.

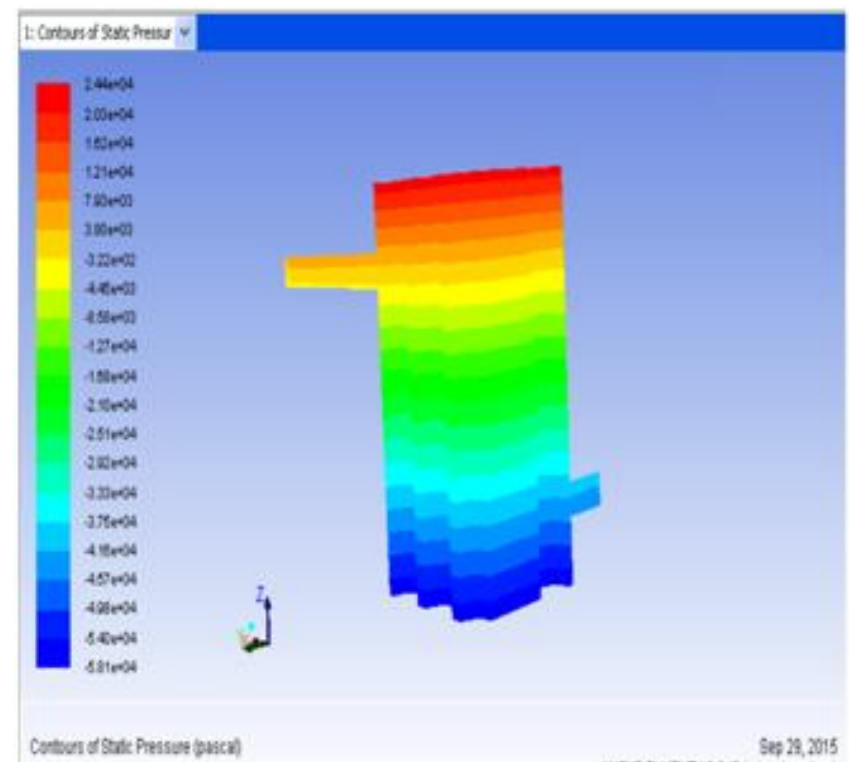

Figure 4.1: Pressure Contour

This experimental setup reveals pressure, temperature and velocity fluctuations of moderator inside the calandria vessel. This may occur in the areas where hot and cold fluid (moderator) interrelates. Moderator velocities vary from boundary of the calandria tube to streamline of the moderator, which may lead to fluctuations of pressure and temperature of the moderator. Due to this, pressure, temperature and velocity profile of the moderator generated. Simulated result on pressure profile shown in the figure 4.1. Pressure profile shows higher pressure at bottom of tank and lower pressure at top of the tank, and this high to low range of pressure forms a pattern.

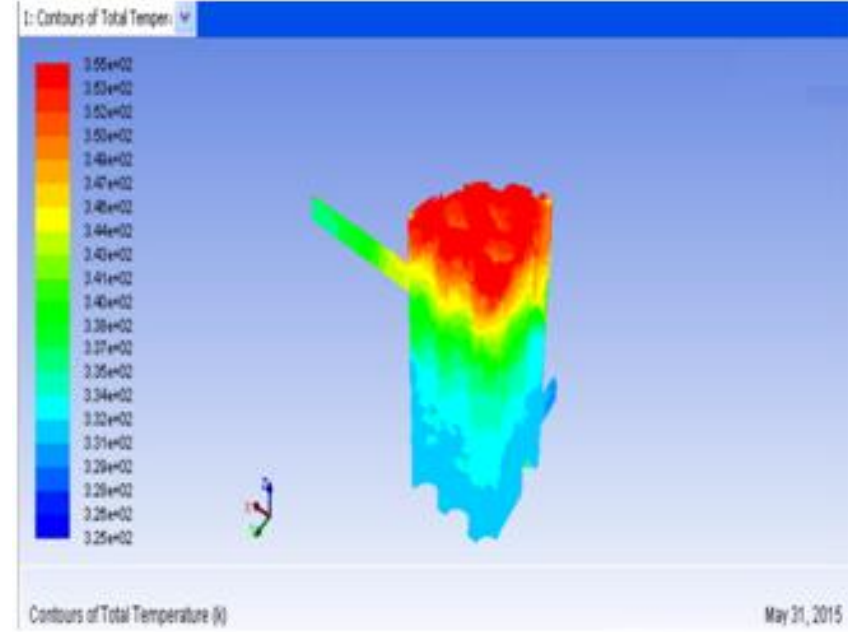

Figure 4.2: Temperature Contour

Simulated result on temperature profile shown in the figure 4.2. The temperature of the moderator is high at the top of the calandria which is approximately $353 \mathrm{~K}$ and bottom of the calandria tank ha $\mathrm{s}$ temperature of $323 \mathrm{~K}$. Simulated results indicate \& conclude, temperature and pressure of moderator is inversely proportional to each parameter.

The highest temperatures are at the top inner zone of the tank and remain unchanged in that zone. As we proceed from the inner to the outer zones of the tank, temperature decreases due to the cold inlet jets which are flowing close to the outer wall. The strongest fluctuations in temperature are observed in the regions where the low velocity hot fluid in the inner parts of the tank, where the flow, mixes with the cold fluid with high velocity flow on the outer regions, referred to as outer jet flow.

The hot region is shifted toward the upward resulting in asymmetric flow in the tank. This occurs due to the competition between buoyancy and momentum forces inside the tank. The high velocity inlet fluid precedes the path of less resistance and flows close to the walls of the tank and outside the tube bundles. Inlets are designed to guide the fluid toward the top of the tank. Therefore, the right and the left cold inlet fluids meet each other somewhere close to the top of the tank. The flow generated by the impingement of these fluids turns downward the core of the tube bundle opposing the upward moving buoyancy flows.

Figure 4.3 shows velocity profile informs direction and magnitude of moderator. Moderator flows with velocity $3.36 \mathrm{e}-03$ and upward direction of calandria vessel in simulated results. The type of flow of moderator in the calandria vessel is steady (velocity is very low) and laminar $(\operatorname{Re}=172)$. 


\section{International Journal of Science and Research (IJSR) \\ ISSN (Online): 2319-7064}

Index Copernicus Value (2013): 6.14 | Impact Factor (2014): 5.611

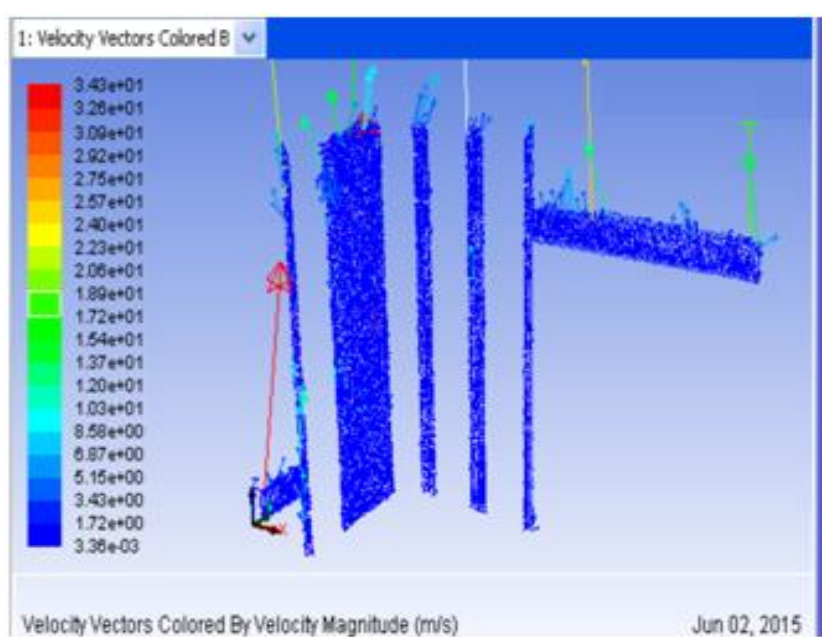

Figure 4.3: Velocity Vector Contour

\section{Conclusion}

Moderator flow in the Calandria vessel studied experimentally and validated by using simulation technique using the student CFD software. Interoperation of pressure Profile, Temperature profile and velocity profile and concluded as follows

\section{Pressure Profile}

Pressure profile shows and interrelates; the pattern of pressure is in ascending from $-7.29 \mathrm{e} 0.4$ to $5.13 \mathrm{e} 0.3$. (Pattern is divided into three regions high-pressure region $(5.13 \mathrm{e} 03$ to $-2.22 \mathrm{e} 04)$, medium pressure region $(-2.61 \mathrm{e} 04$ to $-7.29 \mathrm{e} 04)$ and low-pressure region $(-5.73 \mathrm{e} 04$ to $-7.29 \mathrm{e} 04))$. Pressure varies from the region of $-7.29 \mathrm{e} 0.4$ to $5.13 \mathrm{e} 0.3$. Minimum pressure is $-7.29 \mathrm{e} 0.4$ and maximum pressure is $5.13 \mathrm{e} 0.3$.

Fig. 4.1 shows low-pressure region begins from the top of the calandria vessel and its value is $-7.29 \mathrm{e} 0.4$. bar. And maximum pressure is $5.13 \mathrm{e} 03$ at the bottom of the pressure tank shown by red color. In between of maximum pressure to minimum pressure, a steady and systematic pressure variation generated shown by different colors (fig. 4.1).

\section{Temperature Profile}

Temperature profile varies from $325 \mathrm{k}$ to $355 \mathrm{k}$ (bottom to top upward direction). Temperature profile is not that much steady as compare to pressure profile. It is in descending order from top to bottom in comparison of pressure profile. Hot region is near to top of the vessel. It is very necessary to remove heated moderator to avoid the overheating of core of nuclear power plant. And cold region is only at inlet of temperature $325 \mathrm{k}$.

\section{Velocity Profile \\ Velocity profile shows upward and outward of flow direction of flow. It is Cleary shows the laminar flow as there is not any eddies current. All the velocity vectors are in same direction as shown in figure indicating steady and laminar flow confirming low velocity of flow.}

The above discussion includes all the three profiles. The tank contains cool low-pressure heavy water that surrounds fuel channels. Numerical Simulation of cross flow in staggered tube was performed using FLUENT 14.0 software. SIMPLE algorithm was tested. Viscous Laminar model was used, as Re number is 760 approx.

Data along with velocity and temperature distributions in different planes inside the tank are used to analyze the phenomena occurring inside the tank. The upper region has maximum temperature that is near to outlet and top end shell while low temperature is near to inlet and bottom end shell. As moderator get heated, it is necessary to remove moderator periodically to maintain moderate temperature of calandria vessel so that any accident should not occur. When simulated results are compared with experimental data (which is conducted in laboratory at Bhabha Atomic Research Center Mumbai), the results obtained were found nearly equal.

\section{Future work}

Calandria tank is very complex in terms of geometry and thermal aspects. Inside flow is totally depending on operating condition, heat generation inside the fuel bundles or calandria tubes and extra factors. Simulation showed that the inertia forces and buoyancy are the determining factors in the flow region within the tank. The fluctuations in the temperature and the velocity are powerfully location dependent and vary significantly in cold and hot zones.

Based on this assessment the followings are suggested for the future works:

- Geometry modifications: It is a proper theoretical pilot project to observe the effects of geometry change on the flow and temperature distributions inside the tank. It can include changes in the nozzle locations, nozzle angle and changes in the outlet pipe location.

- Start-up phase modeling: The modeling phase is been prepared and the simulation will start from quiescent flow with zero velocity and temperature all over the domain. In this way we will be able to capture the physics involved in its entire entirety showing the initial phases, which lead to asymmetry in the tank.

\section{Acknowledgement}

I would like to pay attribute to my supervisor, Mukesh Kumar Dhiman for his supervision, advice, and guidance from the very beginning of this project work as well as giving me extraordinary experiences throughout this project work. He is truly more than a scientific supervisor who helped me through all my ups and downs in carrying out this work in BARC Mumbai. He will always be my mentor and I will never forget his role in shaping my future.

My special regards to Dr. M. D. Nadar and Mr. Deepankar Biswas for providing me academic guidance and all support in doing this project work.

I also offer my regards and blessings to Eshita Pal, Nitin Kumar and Sunil Kumarfor getting understand at any type of work and Aboli Khedkar, KalpeshKumar for their companionship and greatly appreciate their patience and good attitude towards me. 
My special thanks go to all my family specially my dear parents, whose affection and support are immeasurable and unforgettable forever. They were beside me whenever I needed and they never gave up in encouraging me to keep going. It would have not been possible to pursue my ME without them.

\section{References}

[1] Teyssedou *, R. Necciari, M. Reggio, F. Mehdi Zadeh and S. Étienne (2014). Moderator flow simulation around calandria tubes of CANDU-6 nuclear reactors. Engineering application of Computational Fluid Mechanics Vol. 8, No. 1, pp. 178-192.

[2] Yoon C, Rhee BW, Bo W, Kim HT, Park J, Min BJ (2006). Moderator analysis of wolsong units $2 / 3 / 4$ for the $35 \%$ reactor inlet header break with a loss of emergency core cooling injection. Journal of Nuclear Science and Technology 43:505-513.

[3] S.S. Bajaj, A.R. Gore (2006). The Indian PHWR. Nuclear Engineering and Design 236 701-722.

[4] R.K. Sinha, A. Kakodkar (2006). Design and development of the AHWR - the Indian thorium fuelled innovative nuclear reactor. Nuclear Engineering and Design 236 683-700.

[5] Deepankar Biswas, Deb Mukhopadhyay, Onkar S Gokhale and M.D. Nadar (2014). Radiation Heat Transfer Code Development in Nuclear Power Plant. International Journal of Latest Trends in Engineering, Science and Technology Vol. 1, Issue 5, pp. 50-52

\section{Author Profile}

Ankul Pratap Singh received his B.Tech degree in Mechanical Engineering from Ishwarchand Vidya Sagar Institute of Technology; Mathura U.P. India affiliated to Uttar Pradesh Technical University, Lucknow UP India. He is pursuing Master of Engineering in Thermal Engineering from, Pillai Institute of Information Technology, Engineering, Media Studies \& Research New Panvel Maharashtra; India affiliated to Mumbai University, Mumbai Maharashtra, India. He is currently working with Blast Carboblocks Pvt. Ltd. Vashi, Navi Mumbai Maharashtra, India as International Business Development and Design Engineer. 